\title{
About The Same
}

National Cancer Institute

\section{Source}

National Cancer Institute. About The Same. NCI Thesaurus. Code C71687.

In an unchanged or similar condition. 